\title{
DEPENDENCIA EMOCIONAL Y VIOLENCIA DE PAREJA EN USUARIAS DEL PRIMER NIVEL DE ATENCIÓN
}

\section{EMOTIONAL DEPENDENCE AND INTIMATE PARTNER VIOLENCE IN FIRST LEVEL HEALTHCARE USERS}

Olga A. Rosas-Muñoz' , Cíbeles Arrambí-Díaz ${ }^{2 *}$, Izkra J. Luna-Bernal' y Rafael Lugo-Valenzuela ${ }^{3}$

'Unidad Médica Familiar 1; ${ }^{2}$ Unidad de Cuidados Intensivos de Quemados, Hospital de Especialidades 2; ${ }^{3}$ Unidad Médica Familiar 67. Instituto Mexicano del Seguro Social, Ciudad Obregón, Sonora, México

RESUMEN: Antecedentes: La violencia hacia la mujer es una infracción a sus derechos humanos y un problema grave de salud pública; se considera el tipo más común de agresión, afecta al 30\% de las mujeres en el mundo. Objetivo: Determinar la correlación entre la dependencia emocional y la violencia de pareja, en mujeres derechohabientes del primer nivel de atención. Material y métodos: Estudio observacional de correlación, donde se incluyó a mujeres entre los 20 y 50 años, con relación de pareja mayor a seis meses; se aplicó el cuestionario de dependencia emocional (CDE) y la Encuesta de Ortega-Ceballos, ambos instrumentos con un índice de confiabilidad alfa de Cronbach de 0.91 y 0.95 respectivamente; se determinó la chi cuadrada de Pearson y la correlación rho de Spearman. Resultados: De 76 pacientes, predominaron las de 30 años o más (61.8\%), de ellas obtuvimos violencia psicológica en un $15.8 \%$, seguida de violencia física en un $2.6 \%$ y sexual en un $1.3 \%$, asociado a dependencia emocional con $\mathrm{p}=0.0001$, y correlación positiva con rho de Spearman de 0.67. Conclusiones: Existe una alta correlación entre dependencia emocional y violencia de pareja en mujeres, y se confirma que la primera se comporta como factor de riesgo para la violencia.

Palabras clave: Dependencia emocional. Violencia de pareja. Atención primaria.
ABSTRACT: Background: Violence against women is an infringement of their human rights and a serious public health problem; is considered the most common type of aggression, affecting $30 \%$ of women worldwide. Objective: To determine the correlation between emotional dependence and intimate partner violence in women patients at the first level of care. Material and methods: Correlational observational study which included women between 20 and 50 years of age, with a relationship of more than 6 months; the emotional dependence questionnaire (CDE) was applied, as well as the Ortega-Ceballos survey, both with a Cronbach's alpha reliability index of 0.91 and 0.95 respectively; Pearson's Chi Square and Spearman's Rho correlation were determined. Results: Of 76 patients, those aged 30 years or more $(61.8 \%)$ predominated, of which we obtained psychological violence $15.8 \%$, followed by physical violence $2.6 \%$ and sexual violence $1.3 \%$, associated with emotional dependence with a $\mathrm{p}=0.0001$, and positive correlation with Rho Spearman's 0.67 . Conclusions: There is a high correlation between emotional dependence and partner violence in women, and it is confirmed that the former behaves as a risk factor for violence.

Keywords: Emotional dependence. Intimate partner violence. Primary care.
Correspondencia:

*Cíbeles Arrambí-Díaz

E-mail: cibelesarrambi @ hotmail.com
Fecha de recepción: 25-03-2021

Fecha de aceptación: 23-08-2021
Disponible en internet: 24-02-2022

Rev Mex Med Fam. 2022;9:5-11 DOI: 10.24875/RMF.21000034

2007-9710 / (C) 2021 Federación Mexicana de Especialistas y Residentes en Medicina Familiar. Publicado por Permanyer. Este es un artículo open access bajo la licencia CC BY-NC-ND (http://creativecommons.org/licenses/by-nc-nd/4.0/). 


\section{INTRODUCCIÓN}

La Organización Mundial de la Salud revela que la violencia de pareja es el tipo más común de agresión contra la mujer y afecta al 30\% de las mujeres en el mundo. En América el 29.8\% de las mujeres ha sido víctimas de violencia física y/o sexual ejercida por parte de su pareja ${ }^{1}$. La violencia a la mujer es un problema grave de salud pública. El 38\% del total de homicidios femeninos se debe a la violencia conyugal $^{1}$, situación que se produce en todos los países, culturas y niveles sociales sin excepción ${ }^{2}$.

La definición propuesta por Heise y García-Moreno ${ }^{3}$, desarrollada y ampliada por Butchart, García-Moreno y Mikton", considera a la violencia de pareja como un tipo de violencia interpersonal y la definen como cualquier comportamiento que dentro de una relación íntima o de pareja causa daño físico, psíquico o sexual, que incluye agresiones físicas (abofetear, golpear con los puños o con cualquier parte del cuerpo, golpes con objetos, empujones, estrangulamiento, etc.), relaciones sexuales forzadas y otras formas de coacción sexual, maltrato psicológico (intimidación, denigración, humillación constante, insultos, gritos, indiferencia, etc.), comportamientos dominantes (aislarla de su familia y amistades, vigilar sus movimientos, restringir su acceso a la información o asistencia, etc.) e incluso la muerte ${ }^{4}$.

En México, según la Encuesta nacional sobre la dinámica de la relación en los hogares (ENDIREH 2016), el 43.9\% de las mujeres de 15 años en adelante ha sufrido algún tipo de violencia por parte de su pareja. En cuanto al nivel educativo, los resultados de encuestas previas muestran que el $18.8 \%$ cuenta con educación superior, el $28.4 \%$ con secundaria, un $24.6 \%$ con primaria y el $21.3 \%$ con educación media superior, reportando el $6.8 \%$ sin estudios, encontrando que el $43.2 \%$ de las mujeres son económicamente activas, y el $42.2 \%$ se dedica a quehaceres del hogar ${ }^{5}$.
Tras analizar la magnitud de la violencia de pareja, resulta importante identificar los factores etiológicos, y en gran proporción estos corresponden a los ubicados a nivel individual, como edad, sexo, escolaridad y características de personalidad, entre otros ${ }^{6}$; algunos estudios han mostrado que las mujeres con baja escolaridad tienen más riesgo de presentarla, esto indica que el nivel educativo tiene un efecto protector ${ }^{7}$. Otro factor es el maltrato o abuso que se experimenta durante la infancia y que se inflige por parte de los padres, ya que este tipo de conducta tiende a perpetuarse a lo largo del desarrollo de las mujeres ${ }^{8}$. Las variables psicológicas individuales son otro factor, en este contexto está la dependencia emocional a la pareja, el apego en relación con los componentes emocionales, cognitivo, conductual y social, dando lugar a sentimientos como la pertenencia, la seguridad y la autoestima?

Castelló define la dependencia emocional como «un patrón persistente de necesidades emocionales insatisfechas que se intentan cubrir de manera desadaptativa con otras personas» ${ }^{10}$. Y es en esta necesidad, el miedo a la pérdida, al abandono y a muchos otros aspectos tales como la justificación de las infidelidades y agresiones de su pareja, que cancelan procesos legales, incumplen las órdenes judiciales de alejamiento, regresan con el agresor creyendo que es posible que sus parejas tomen conciencia y cambien de actitud, entre otros ${ }^{11,12}$. El presente estudio surge de la necesidad de analizar la relación entre la dependencia emocional y la violencia conyugal en el primer nivel de atención, y buscar las causas que vinculen estas variables.

\section{MATERIAL Y MÉTODOS}

Previa evaluación y posterior a ser aceptado por el Comité de Ética e Investigación de la Unidad Médica Familiar 1, en Ciudad Obregón, Sonora, se realizó un estudio observacional de correlación, se calculó la muestra con la fórmula para determinar correlación simple en un grupo, y el tipo de muestreo fue no probabilístico 
por casos consecutivos. El estudio se llevó a cabo de agosto a octubre de 2020. Se realizó en mujeres de 20 a 50 años, a las cuales se les aplicaron las encuestas mientras se encontraban en la sala de espera de la unidad, y debían cumplir los criterios de selección: ser derechohabiente, tener una relación de pareja mayor a seis meses, firmar carta de consentimiento y que no tuvieran antecedentes de enfermedad psicológica y/o psiquiátrica, excluyendo también a mujeres gestantes o puérperas. Las variables que buscamos fueron: edad, ocupación, estado civil, escolaridad, tiempo de relación en pareja, violencia de pareja y dependencia emocional.

Los instrumentos que se utilizaron fueron dos:

- El primer instrumento está basado en el ISA (Index of Spouse Abuse), así como en la SVAWS (Severity of Violence Against Women Scale) (Marshall, 1992), conocida como la Encuesta de Ortega Ceballos, et al. ${ }^{13,14}$. Este instrumento fue validado en México en el 2007 por dicho autor con un estudio que determina violencia de pareja en trabajadoras del Instituto Mexicano del Seguro Social (IMSS). Se realizó en la ciudad de Morelos en el 2006 ${ }^{14}$. En él se evalúan los actos de violencia en los últimos doce meses; cuenta con 35 reactivos, donde se conceptualiza violencia física con 17 preguntas, violencia psicológica con 14 y violencia sexual con 4, con opciones de respuesta tipo Likert. El índice de confiabilidad alfa de Cronbach para todo el instrumento es de $0.95^{15}$.

- El segundo es el cuestionario de dependencia emocional (CDE) (Lemos y Londoño, 2006), que fue validado en México por Laca Arocena y Mejía Caballeros en el 2017, con la investigación psicológica del Estado de Colima ${ }^{16}$. Este instrumento está compuesto por 23 ítems presentados en forma de afirmaciones, donde mide seis dimensiones o factores de dependencia emocional en relaciones de más de seis meses, donde seis preguntas son destinadas a valorar la ansiedad a la separación, cuatro para la expresión afectiva, cuatro para modificación de planes, tres que valoran el miedo a la soledad, tres más a expresión limite y finalmente dos preguntas a búsqueda de atención, el índice de confiabilidad alfa de Cronbach para todo el instrumento es de $0.91^{15,16}$.

\section{ANÁLISISESTADÍSTICO}

El análisis univariante se realizó en variables cualitativas, frecuencia y porcentaje, y para las variables cuantitativas se determinó medidas de tendencia central (media, mediana y moda). En el análisis bivariante se realizó la prueba de chi cuadrada para variables no paramétricas de escala nominal, tomando una significancia estadística de $\mathrm{p}<0.05$.

$\mathrm{Y}$ finalmente para determinar correlación, se usó el Coeficiente de correlación Rho de Spearman en las variables cualitativas con distribución libre.

\section{RESULTADOS}

De una muestra de 76 pacientes, se obtuvo que 47 de ellas fueron de 30 años o más $(61.8 \%)$ y 29 (38.2\%) menores de 30. En la variable ocupación, observamos que la mayoría de las pacientes eran empleadas (65.8\%), amas de casa el $21.1 \%$ y estudiantes el $13.2 \%$. En relación con el estado civil encontramos que predominó el de casadas (52.6\%), seguido de la unión libre (39.5\%) y en menor frecuencia solteras (7.9\%).

En cuanto a la escolaridad podemos describir que el nivel profesional fue el predominante $(32,42.1 \%)$, seguido del bachillerato $(28,36.8 \%)$, secundaria (12, $15.8 \%)$ y primaria $(4,5.3 \%)$. Con respecto al tiempo de relación en pareja, se obtuvo que de un año o más fue el predominante $(65$, $85.5 \%)$ y solo 11 (14.5\%) fueron menor de un año. En la variable dependencia emocional encontramos: pacientes sin dependencia 
emocional un $80.3 \%(\mathrm{n}=61)$ y un $19.7 \%$ (n =15) presentó dependencia.

La dependencia emocional y sus diferentes tipos muestra una mayor presentación, para pacientes sin dependencia $(60,78.9 \%)$, seguido de dependencia normal $(11,14.5 \%)$ $\mathrm{y}$ en menor frecuencia las pacientes altamente dependientes $(5,6.6 \%)$. La variable violencia de pareja tiene una presentación alta para mujeres sin violencia $81.6 \%(\mathrm{n}=$ $62)$, seguida de con violencia $18.4 \%(n=14)$. En cuanto a los tipos de violencia infligida a la pareja, se muestra un predominio para sin violencia $(61,80.3 \%)$, seguido de violencia psicológica $(12,15.8 \%)$ violencia física $(2,2.6 \%)$ y violencia sexual $(1,1.3 \%)$ (Tabla 1). La prueba cruzada para asociación entre dependencia emocional y violencia de pareja muestra una chi cuadrada de Pearson con valor de 0.0001 , con una estimación de riesgo de 16.8 e intervalo de confianza del $95 \%$ inferior de 4.2 y superior de 66.7 (Tabla 2), obteniendo además una correlación positiva entre dependencia emocional y violencia de pareja con una rho de Spearman de 0.67 ( $\mathrm{p} \leq 0.01$ ), interpretándose como una alta fuerza de correlación entre ellas (Tabla 3 ).

\section{DISCUSIÓN}

Los hallazgos del presente estudio de correlación entre dependencia emocional y violencia de pareja indican que aproximadamente dos de cada diez mujeres presentan eventos de violencia infligida por parte de su pareja, cuya prevalencia fue del $18 \%$, la cual es muy similar a la que se reportó en un estudio previo realizado en atención primaria, Salvador-Jurado ${ }^{13}$.

Respecto a la tipología de violencia de pareja, se encontró que el $15.8 \%$ de las mujeres presentaron violencia psicológica, seguido del $2.6 \%$ con violencia física y en menor proporción violencia sexual con $1.3 \%{ }^{17}$.

Se han llevado a cabo investigaciones que analizan factores que influyen en la etiología de la violencia de pareja. El modelo ecológico de Bronfenbrenner incluye una serie de elementos que se ubican en diferentes estadios de la vida, que actúan como
Tabla 1. Frecuencia de variables cualitativas

\begin{tabular}{|c|c|c|c|}
\hline Variable & & $\begin{array}{c}\mathrm{N}: \\
\text { frecuencia }\end{array}$ & $\%$ \\
\hline \multirow[t]{3}{*}{ Estado civil } & Soltero & 6 & 7.9 \\
\hline & Unión libre & 30 & 39.5 \\
\hline & Casado & 40 & 52.6 \\
\hline \multirow[t]{4}{*}{ Escolaridad } & Primaria & 4 & 5.3 \\
\hline & Secundaria & 12 & 15.8 \\
\hline & Bachillerato & 28 & 36.8 \\
\hline & Profesional & 32 & 42.1 \\
\hline \multirow{2}{*}{$\begin{array}{l}\text { Tiempo } \\
\text { de relación } \\
\text { en pareja }\end{array}$} & Menor de 1 año & 11 & 14.5 \\
\hline & 1 año o más & 65 & 85.5 \\
\hline \multirow[t]{3}{*}{ Ocupación } & Ama de casa & 16 & 21.1 \\
\hline & Empleada & 50 & 65.8 \\
\hline & Estudiante & 10 & 13.2 \\
\hline \multirow{3}{*}{$\begin{array}{l}\text { Dependencia } \\
\text { emocional }\end{array}$} & Sin dependencia & 60 & 78.9 \\
\hline & Dependencia normal & 11 & 14.5 \\
\hline & $\begin{array}{l}\text { Altamente } \\
\text { dependiente }\end{array}$ & 5 & 6.6 \\
\hline \multirow{4}{*}{$\begin{array}{l}\text { Violencia de } \\
\text { pareja }\end{array}$} & Sin violencia & 61 & 80.3 \\
\hline & Violencia psicológica & 12 & 15.8 \\
\hline & Violencia física & 2 & 2.6 \\
\hline & Violencia sexual & 1 & 1.3 \\
\hline
\end{tabular}

determinantes o factores de protección ante las situaciones de violencia de pareja. Esta aproximación estudia la manera en que los individuos interactúan con el medio ambiente mediante un conjunto de estructuras concéntricas (microsistema, mesosistema, exosistema y macrosistema) para explicar las raíces biográficas, psicológicas, familiares, relacionales, comunitarias, sociales y ambientales de la reproducción de la violencia de pareja ${ }^{8,17}$.

Una gran proporción de los factores de riesgo que se asocian a la violencia de pareja corresponde a situaciones ubicadas en el nivel individual. En el presente estudio se encontró que la edad también es un factor de riesgo para violencia, pues a mayor edad de la mujer, mayor riesgo, lo cual difiere del estudio de Ortega-Ceballos et al., aunque no es estadísticamente significativa ${ }^{14}$. En cuanto a la educación, encontramos que la baja 
Tabla 2. Análisis bivariante entre violencia de pareja y otras variables

\begin{tabular}{|c|c|c|c|c|c|}
\hline \multirow[t]{2}{*}{ Variable } & \multirow[t]{2}{*}{ Características } & \multicolumn{2}{|c|}{ Violencia de pareja } & \multirow{2}{*}{$\begin{array}{c}\text { OR } \\
\text { IC } 95 \%\end{array}$} & \multirow[t]{2}{*}{ p } \\
\hline & & Con & $\operatorname{Sin}$ & & \\
\hline \multirow[t]{2}{*}{ Edad } & Menor de 30 & 5 & 24 & $\begin{array}{c}0.8 \\
(0.2-2.9)\end{array}$ & 0.8 \\
\hline & 30 años o más & 9 & 38 & & \\
\hline \multirow[t]{2}{*}{ Estado civil } & Soltero & 7 & 29 & $\begin{array}{c}0.8 \\
(0.2-2.9) \\
\end{array}$ & 0.8 \\
\hline & Casado & 7 & 33 & & \\
\hline \multirow[t]{2}{*}{ Ocupación } & No empleada & 3 & 13 & $\begin{array}{c}1 \\
(0.2-4.2)\end{array}$ & 0.9 \\
\hline & Empleada & 11 & 49 & & \\
\hline \multirow[t]{2}{*}{ Escolaridad } & Básica & 4 & 12 & $\begin{array}{c}1.6 \\
(0.4-6.2) \\
\end{array}$ & 0.4 \\
\hline & Media superior & 10 & 50 & & \\
\hline \multirow[t]{2}{*}{ Tiempo de relación } & Menor de 1 año & 1 & 10 & $\begin{array}{c}0.4 \\
(0.04-3.4)\end{array}$ & 0.3 \\
\hline & 1 año o más & 13 & 52 & & \\
\hline \multirow[t]{2}{*}{ Dependencia emocional } & Con dependencia & 9 & 6 & $\begin{array}{c}16.8 \\
(4.2-66.7) \\
\end{array}$ & 0.0001 \\
\hline & Sin dependencia & 5 & 56 & & \\
\hline
\end{tabular}

OR: odds ratio; IC 95\%: intervalo de confianza al 95\%; p: significancia estadística.

Tabla 3. Correlación entre violencia de pareja y variables

\begin{tabular}{|l|l|c|c|c|c|c|c|}
\hline \multicolumn{2}{|c|}{} & $\begin{array}{c}\text { Dependencia } \\
\text { emocional }\end{array}$ & Edad & $\begin{array}{c}\text { Tiempo de } \\
\text { relación en } \\
\text { pareja }\end{array}$ & Ocupación & Estado civil & Escolaridad \\
\hline $\begin{array}{l}\text { Violencia } \\
\text { de pareja }\end{array}$ & $\begin{array}{l}\text { Coeficiente } \\
\text { de Correlación }\end{array}$ & $0.671^{*}$ & 0.047 & 0.023 & 0.003 & -0.13 & -0.081 \\
\cline { 2 - 8 } & Sig. (bilateral) & 0.000 & 0.685 & 0.841 & 0.982 & 0.238 & 0.489 \\
\cline { 2 - 8 } & N. ${ }^{\circ}$ & 76 & 76 & 76 & 76 & 76 & 76 \\
\hline
\end{tabular}

Sig.: significancia; N. 0 : número total de encuestas.

*Nivel de dependencia: 0-0.2 leve, 0.3-0.5 moderada, 0.6-0.8 alta, 0.8 muy alta.

escolaridad tiene 1.6 probabilidades de tener violencia pero sin significativa estadística, a diferencia de otros investigadores los cuales encontraron que las mujeres con baja escolaridad sí tienen más riesgo de presentar violencia de pareja ${ }^{18}$. El nivel educativo tiene un efecto protector, ya que se vincula con una mayor capacidad no solo económica, sino también para resolver los conflictos en la relación de pareja, ya que le confiere mayor poder y jerarquía en relación con los estándares sociales ${ }^{19}$.
La ocupación tampoco fue significativa y sin correlación (Tabla 3), aunque otros autores han determinado que la mujer con autonomía para la toma de decisiones tiene una probabilidad baja de presentar experiencias de abuso físico y sexual, en comparación con las mujeres desempleadas, cuyo comportamiento es controlado por su pareja. Este hallazgo se fundamenta en la teoría de intercambio social, que señala que la autonomía y la toma de decisiones permiten equilibrar el control y el poder de 
la relación de pareja ${ }^{20}$. El estado civil tampoco tiene diferencia, aunque en estudio previo $^{21}$ muestra que las mujeres casadas presentan mayor porcentaje de violencia que los solteros, aunque refieren que la idealización y la falta de tiempo en pareja de los solteros o recién casados es mínima para percibir grandes discrepancias. Por el contrario, otros consideran un factor de riesgo importante para violencia de pareja el estar en unión libre (Ortega-Ceballos et al. $)^{14}$.

Por último, nuestro estudio sugiere que existe una relación estadísticamente significativa entre la variable dependencia emocional y la variable violencia de pareja, con correlación alta, lo cual concuerda con estudios previos ${ }^{22,23}$.

De esta forma, una mujer con dependencia emocional experimenta elevados niveles de miedo al pensar que su relación de pareja pueda terminar. El miedo como respuesta a la violencia puede funcionar de forma ambivalente. Por un lado, puede ser una forma de afrontamiento activo si se asocia a la huida y evitación, alejando a las mujeres de la situación de violencia. Por otro lado, si el miedo aparece en situaciones de violencia continuada puede suponer un aprendizaje y habituación a ella ${ }^{23}$.

Desde este punto de vista, consideramos importante aportar alternativas de intervención psicológica en la prevención y tratamiento de mujeres víctimas de violencia de pareja. Así, las actividades de prevención y promoción, principalmente en la población adolescente y joven, han de incidir en las consecuencias de depender emocionalmente de la pareja, y cómo esta característica permite tolerar actitudes y conductas de violencia, justificándose estos comportamientos por el supuesto "amor incondicional que se ha de tener a la pareja» (entre otras creencias individuales y sociales), lo cual precipita nuevamente ciclos de violencia cada vez más severos que probablemente, si no se hace algo al respecto, lleven a otro caso más de feminicidio. Queda pendiente seguir investigando más sobre la relación entre dependencia emocional y violencia de pareja en muestras más grandes, de diferentes procedencias y desde otro enfoque de investigación como cualitativo o mixto.

\section{CONCLUSIÓN}

Se obtuvo que existe una buena correlación entre las variables de estudio, confirmando que la dependencia emocional se comporta como un factor de riesgo para la presencia de maltrato por parte de la pareja.

\section{AGRADECIMIENTOS}

A todas las mujeres que han sido víctimas de algún tipo de violencia, así como a los profesionales del centro de atención primaria de salud que accedieron a participar y colaborar en el estudio.

\section{FINANCIAMIENTO}

La presente investigación no ha recibido ninguna beca especifica de agencias de los sectores públicos, comercial o con ánimo de lucro.

\section{CONFLICTO DE INTERESES}

Los autores declaran no tener conflicto de intereses.

\section{RESPONSABILIDADESÉTICAS}

Protección de personas y animales. Los autores declaran que para esta investigación no se han realizado experimentos en seres humanos ni en animales.

Confidencialidad de los datos. Los autores declaran que han seguido los protocolos de su centro de trabajo sobre la publicación de datos de pacientes.

Derecho a la privacidad y consentimiento informado. Los autores han obtenido el consentimiento informado de los pacientes y/o sujetos referidos en el artículo. Este documento obra en poder del autor de correspondencia. 


\section{BIBLIOGRAFÍA}

1. García-Moreno C, Henrica A, Watts C, Ellsberg M, Heise L. Estudio multipaís de la OMS sobre salud de la mujer y violencia doméstica contra la mujer [Internet]. Ginebra: Organización Mundial de la Salud; 2005 [consultado: junio 2019]. Disponible en: https:// www.who.int/gender/violence/who_multicountry_study/summary report/chapter1/es

2. Dubugras S, Guevara B. Personalidad de mujeres víctimas de violencia doméstica: una revisión sistemática da literatura. Contextos Clínicos. 2013;6(2):106-16.

3. Heise L. Violencia contra la mujer. La cara oculta de la salud [Internet]. Washington: Programa Mujer, Salud y Desarrollo, Organización Panamericana de la Salud; 1994 [actualizado: octubre 2001, consultado: mayo 2019]. Disponible en: http://genero.bvsalud.org/dol/docsonline/0/4/040-gph4.PDF

4. Butchart A, García-Moreno C, Mikton C. Prevención de la violencia sexual y violencia infligida por la pareja contra las mujeres: qué hacer y cómo obtener evidencias [Internet]. Washington: Organización Panamericana de la Salud; 2011 [consultado: junio 2019]. Disponible en: https://oig.cepal.org/sites/default/files/9789275316351_spa.pdf

5. Instituto Nacional de Estadística y Geografía. Encuesta Naciona sobre la Dinámica de las Relaciones en el Hogar, ENDIREH 2016 [Internet]. México: INEGl; 2016 [consultado: junio 2019]. Disponible en: https://inegi.org.mx/contenidos/programas/endireh/2016/doc/endireh2016_guia_autocuidado.pdf

6. Instituto Nacional de estadística y Geografía. Resultados de la Encuesta Nacional sobre la Dinámica de las Relaciones en los Hogares, ENDIREH 2016. [En internet]. México: INEGI; 2016 [consultado: junio 2019]. Disponible en: https://www.gob. $\mathrm{mx} / \mathrm{cms} / \mathrm{uploads} / \mathrm{attachment/file/318425/ENDIREH2016}$ BoletinPrensa2017_INEGI.PDF

7. Amor P, Echeburúa E. Claves psicosociales para la permanencia de la víctima en una relación de maltrato. Clínica Contemporánea. 2010;1(2):97-104.

8. Rivera-Rivera L, Allen B, Chávez-Ayala R. Ávila-Burgos L. Abuso físico y sexual durante la niñez y revictimización de las mujeres mexicanas durante la edad adulta. Salud Publica Mex. 2006;48(2):268-78

9. Bowlby J. Vínculos afectivos: formación, desarrollo y pérdida. 6 a $^{\text {a }}$ ed. Madrid, España: Morata; 1986.
10. Castelló J. Dependencia emocional: Características y tratamien-

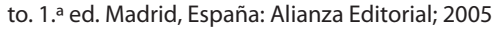

11. Castelló J. La superación de la dependencia emocional. Cómo impedir que el amor se convierta en un suplicio. 1. a ed. Málaga, España: Ediciones Corona Borealis; 2012.

12. Villegas $M$, Sánchez N. Dependencia emocional en un grupo de mujeres denunciantes de maltrato de pareja. Revista Textos \& Sentidos. 2013;7:10-29.

13. Salvador-Jurado $L$, Jiménez-Báez $M$, Rovira-Alcocer $G$, VitalHernández O, Pat-Epadas F. Violencia de pareja: tipo y riesgo en usuarias de atención primaria de salud en Cancún, Quintana Roo, México. Aten Primaria. 2017;49(8):465-72

14. Ortega-Ceballos PA, Mudgal J, Flores Y, Rivera-Rivera L, DíazMontiel JC, Salmerón J. Determinantes de violencia de pareja en trabajadoras del IMSS Morelos. Salud Publica Mex. 2007;49(5):357-66.

15. Aiquipa J. Diseño y validación del inventario de dependencia emocional-IDE. Rev Invest Psicol. 2012;15(1):133-45.

16. Laca FA, Mejía JC. Dependencia emocional, consciencia del presente y estilos de comunicación en situaciones de conflicto con la pareja. Enseñanza Invest Psicol. 2017;22(1):66-75.

17. Jaen-Cortés $\mathrm{Cl}$, Aragón $\mathrm{SR}$, Amorin de Castro EF, Rivera Rivera L. Violencia de pareja en mujeres: prevalencia y factores asociados. Acta de Investigación Psicológica. 2015;5(3):2224-39.

18. Ávila-Burgos L, Valdez-Santiago R, Barroso-Quiab A, Híjar M, Rojas $\mathrm{R}$, del Río-Zolezzi A. Prevalencia y factores asociados a violencia de pareja en usuarias de servicios públicos de salud en México: un análisis comparativo. Rev Invest Clin. 2014;66(1):45-58.

19. González H, Fernández de Juan T. Género y maltrato: violencia de pareja en los jóvenes de Baja California. Estud Front. 2010;11(22):97-128.

20. Antai D. Controlling behavior, power relations within intimate relationships and intimate partner physical and sexual violence against women in Nigeria. BMC Public Health. 2011;11:511.

21. Cáceres $A$, Cáceres J. Violencia en relaciones íntimas en dos etapas evolutivas. Int J Clin Health Psychol. 2006;6(2):271-84.

22. Aiquipa J. Dependencia emocional en mujeres víctimas de violencia de pareja. Revista de Psicología. 2015;33(2):412-37.

23. Echeburua E, Pedro J, Amor y Paz C. Mujeres maltratadas en convivencia prolongada con el agresor: variables relevantes. Acción Psicológica. 2002;2:135-50. 